\title{
The Challenges, Innovation and Development of University Broadcasting in the Context of Convergence Media: To Jiangsu University Broadcast Station as an Example
}

\author{
He Wei \\ Propaganda Department of Party Committee, Jiangsu University, Zhenjiang, China
}

Email address:

441883649@qq.com

To cite this article:

He Wei. The Challenges, Innovation and Development of University Broadcasting in the Context of Convergence Media: To Jiangsu University Broadcast Station as an Example. Education Journal. Vol. 6, No. 6, 2017, pp. 215-218. doi: 10.11648/j.edu.20170606.18

Received: November 30, 2017; Accepted: December 15, 2017; Published: January 4, 2018

\begin{abstract}
With the rapid development of new online media, the traditional media has been greatly impacted. Being an important tool of campus propaganda, university broadcasting faces the urgent problem that how to go with the stream of convergence media. The article analyzes the present situation and existing problems of campus radio in colleges and universities and discusses the opportunities and challenges that campus broadcasting faces in the context of convergence media. Based on that, the article puts forward suggestions on the innovation and development of university campus.
\end{abstract}

Keywords: Convergence Media, Colleges and Universities, Campus Radio

\section{Introduction}

University broadcasting, the forefront of the construction of campus culture, is the important media which can improve the ideological and political education in universities and colleges. With the development of the new network media, campus radio stations face unprecedented challenges. It is very important and urgent for campus radio stations to pass the bottleneck period, know the idea of new media in an active, rational and scientific way and create personalized broadcast production by integrating with new media.

\section{The Present Situation and Problems of University Broadcasting}

\subsection{The Overall Quality of Announcers Needs Further Enhance}

The quality of radio program depends largely on the quality of announcers. The main announcers of campus radio are students who mainly rely on self-training and self-learning, rather than rigorous, systematic and professional training. Therefore, there are knowledge gaps between pronunciation skills and professional knowledge. Campus radio is broadcast in real time, so during broadcasting, there will exist blowing into microphone, apex tone, slur words subenergetic phonation and other problems, which influences the broadcast effect [1].

\subsection{Radio Program Production Mechanism Is Unsound}

In terms of general broadcast production, a program production chain often includes three mechanisms: the incentive mechanism for the beginning of the program, the guarantee mechanism during the broadcasting and the evaluation mechanism after the program. Three types of mechanisms constitute a system chain, effectively activating the program's innovation, stability and development[2]. However, due to some factors like the backward ideas about university broadcasting, there are just two links, examination and broadcasting, in most university broadcasting. Even when the program is finalized, there is just the broadcast link. As the result, the program lacks of innovation, guarantee and evaluation, which can affect the quality of the program and good reception of audience [3].

\subsection{Underutilizing the Advantages of Network New Media Technology}

The appearance of any kind of new media is at the expense of oppressing the old one. At present, the rapid development of the new media technologies and numerous new applications 
bring unprecedented impact to the traditional media such as broadcasting. In the era of new online media, campus broadcasting cannot make timely use of this large platform, lacks interaction and cooperation with it. Therefore, the discourse power of broadcasting media is declining [4]. The only ways to develop traditional media like broadcasting are to strengthen the integration of campus radio with new online media, to make full use of the advantages of new online media technologies and to enhance the breadth and depth of broadcasting.

\subsection{Limited Broadcast Time and a Single Communication Channel}

Because of its own limitations, campus radio must strictly control the broadcasting time. There exist many problems, such as limited broadcast time, fixed play time, limited program content and only one time. So, it cannot meet the individualized listening needs of teachers and students. In addition, broadcast time coincides with the peak time. After class, eating and other noisy sound come along, the transmission effect is greatly affected. At present, radio and television broadcasting in colleges and universities still adopt the method of cable broadcasting, and the density and breadth of coverage of broadcasting circuits directly determine the effect of campus broadcasting. Most campus radio equipment is old, outdated and the radio coverage is low, which influence the breadth of broadcasting. The upgrading of hardware facilities and the development of diversified channels of communication become urgent problems to be solved in promoting radio and television propaganda [5].

\section{The Opportunities and Challenges of Campus Broadcasting in the Context of Convergence Media}

\subsection{Opportunities}

(1) The program material access channel is changing. With the rapid development of new online media, information acquisition become more convenient and diverse. Past broadcast material was often obtained through submissions from other departments or through field interviews. However, nowadays, it is faster and easier to access information through the new web platform.

(2) The program broadcast channels are more abundant. With the development of science and technology, radio is no longer just on the radio, but more internet platforms provide a live channel for live broadcasting, such as Qingting FM, Penguin FM. In addition, a variety of video broadcast software makes the radio become very impressive.

(3) The communication platforms for audience are more diversified. Not only working behind the scenes, anchors can also communicate with listeners through social platforms. In this way, they can understand listeners' preferences, absorb opinions and suggestions and then they can continuously improve the quality of the programs [6].

\subsection{Challenges}

(1) The ability to collect and process information needs to be strengthened. In the context of Convergence Media, the information content is extensive, intricate, and spreads fast. Campus media needs to promote the mainstream culture and seek advantages and avoid disadvantages. What's more, campus media should avoid sensitive and inappropriate news and topics, which requires broadcasters themselves have the ability to collect, filter and process information.

(2) The ability to adapt to new technologies needs to develop. With the development of science and technology, broadcasting can no longer rely on simple microphones. Announcers should fully grasp the most advanced audio editing technologies and methods of equipment. They should also master the use of various new media and make full use of diversified media.

(3) The innovation ability of the program needs to improve. If the program wants to stand out various media and have the high radio station, it must be distinctive. It is unrealistic to only rely on the new media to collect information. That requires broadcasters improve their original capabilities and integrate themselves into their own thinking [7] [8].

\section{The Innovation and Development of Campus Broadcasting in the Context of Convergence Media}

\subsection{Make Full Use of New Online Media Technologies to Enhance Self-Promotion}

New online media has become one of the most important ways for college students to learn, exchange and entertain because of itscharacteristics like convenient communication, diversifiedcarriers, interactive content and virtuality. As a traditional media, campus radio must make full use of the technological advantages of new online media and enhance self-promotion so as to find a chance of survival [9]. According to this feature, the Radio Station of Jiangsu University creates the WeChat public number on the basis of the original traditional broadcasting, uploads the anchor introduction and the in-platform display, and pushes the fine programs and recommendation of new songs every month. By this way, the traditional media can spread better and keep closer contact with teachers and students. In addition to WeChat, the radio station also carries out a lot of self-promotion through multiple channels, such as microblogging, Renren, QQ public number, Baidu Encyclopedia, Qingting FM live online platform and NetEase cloud platform. This method, to a certain extent, makes up for the restrictions of time and space, enhances the freedom and initiative of audience to select information and improves the dissemination effect. At the same time, the integration of radio and new online media provide a viable platform for the exchange of broadcasting programs among various colleges and universities. 


\subsection{The Promotion of the Overall Quality of Campus Announcers}

(1) Strict control of "entrance"is to ensure the quality of announcers from the source. When new students are enrolled in colleges and universities, the radio station finally confirms the candidates after registration, preliminary examination, reexamination and a month training internship. The process is intense with strict requirements. Assessment is mainly around the sound conditions and the ability of Putonghua. During internship period, candidates are required to create a radio broadcast by themselves, which mainly tests their ability of planning programs, program editing manuscripts and post-production.

(2) The campus radio station focuses on training and learning and technically improve announcers' professional quality. Before taking part in the radio station, the Jiangsu University radio station will train every announcer on the audio editing software. From cooledit to Adobe Audition, the radio station constantly update the technologies and enables each announcer to master the new technologies and then ensures the quality of the program.

(3)The campus radio station pays attention to the system construction, the preciseness and the sense of responsibility of announcers. The broadcasting station of Jiangsu University has clear working rules, strict working system, regular meeting system, duty system and leave system. All members must observe those rules and defend the honor of radio station.

(4) The radio station need strengthen team building, strive to create a rigorous, lively, independent and united" working atmosphere and independence" and try to create a team of proactive, united and progressive broadcasters [10].

\subsection{Reform Programs and Improve Program Quality}

In the face of fierce media competition, broadcasting must start from the details to enhance reform and innovation, improve program quality and develop its own competitiveness.

(1) The program design is modularized. Open-air campus radio's broadcast time is limited, so how to hold the attention of passers-by long enough? All programs of Jiangsu University radio station are modularized. Each section is controlled in about 7-8 minutes and each section has a separate header in order to distinguish from different parts. Through modularization, listeners will not only listen to a complete piece of content within the fragmentation time, but broadcast programs will also become clearer.

(2) Collection, edition and broadcast are integrated. At present, many college radio stations separate editing from broadcasting. This system is very convenient for doing news broadcast programs. However, problems can easily emerge in connection work. Each program of Jiangsu University radio station will adopt the integration of editing and broadcasting. The anchor is responsible for collecting data, creating secondly, editing and broadcasting. The integration can helpanchorsunderstand program generally, optimize the program effect and practice the ability of anchors.

(3) The proportion of recorded programs should be increased. Jiangsu University radio station need give priority to record-based and write draft in advance to ensure the broadcast quality.

(4) Program is multiple. Jiangsu University radio station's programinclude news, campus, music, entertainment, culture and combine Chinese and Western, in order to provide students and students rich and colorful campus culture. The radio station also tries some new models such as talk shows, radio play, radio site connection and so on.

\subsection{Strengthen the Interaction with the Audiences and the Internal Exchanges}

Campus radio as a campus media, to strengthen the interaction with the audience is the requirement of both the campus radio itself and university students. In order to improve the listening rate and the program quality, the campus radio must listen to the opinions and comments of the audiences. Such interaction must be established and put into play [11]. In each program, JiangsuUniversity radio station will leave an interactive way to keep abreast of the audience's responses. In addition, there is a fixed interactive song section - "Retain the Innermost Words ". The audience send songs to others via telephone messages, third-party messages and other ways and the announcer broadcast in the process. There is a large live interactive program at weekends - "Voice of Angels", in which the anchor and the audience can achieve real-time interaction through Qingting FM live online platform. While paying attention to the audiences' voices, the radio station also emphasizes internal communication. It makes full use of the regular meeting system to conduct a one-week summary and program review. All broadcasters can learn from each other and share their experiences in broadcasting and programming and they can take as needed to improve together.

\subsection{Reinforce Learning and Improve Themselves}

Jiangsu University radio station maintains cooperation with Zhenjiang Media Group. The group provides announcers summer internship opportunities that the announcers of the campus, to accept more professional technical guidance and practice. Zhenjiang Cultural Group also set up a special student self-made radio music program "private music home," so that the announcer actual combat operations, and effectively enhance professional standards. In addition, radio stations at JiangsuUniversity often hold exchanges with fraternal colleges and face to face understanding of each other's broadcasting work flow, learn from each other and make progress together.

\section{Conclusion}

Campus radio is an indispensable part of university campus culture. Under the rapidly developing new media environment, innovation and breakthroughs should be made only in terms of concept, working mechanism and team building. Campus radio also should give full play to the content advantages of traditional media and the channel advantages of emerging 
media so as to realize complementary advantages and deep integration, establishthe communication platform based on the traditional but not traditional and keep pace with the new media. New media has diverse formats, advanced means and competitiveness, so it will promote the campus radio to achieve its own innovation and development and operate more smoothly.

\section{References}

[1] Xie Zhengyang. Brief Discussion on Broadcast Skills for News Broadcasts[J]. West China Broadcasting TV, 2015-03-25

[2] GuNannan. Analyzing Current Living Situation of Radio Show Studios [J]. China Broadcasts, 2014-01-15

[3] Zhang Luocheng. Analysis of Campus Broadcasting in the New Media Age - Zhejiang Institute of Media as an Example [J] News Sentinel, 2014-11-15

[4] Yang Yufeng. The Development Difficulties and Strategies of College Broadcasting in the New Media Era [J]. Journalism \& Communication, 2014-05-15
[5] Wu Yongman. The New Era of College Broadcasting Development Predicament and Coping Strategies [J]. Journal of Shaoguan University, 2016-07-15

[6] Sun Wenxia. The Role of New Media in Traditional Broadcasting [J]. Voice \& Screen World, 2015-04-01

[7] Zhu Zhengan. TheDevelopment and Strategies of Broadcasting in New Media Times [J]. Journal of Southwest Jiaotong University (Social Sciences Edition), 2013-01-15

[8] Bai Yichen. Current Situation and Development of College Campus Media[J]. Journal of News Research, 2016-12-10

[9] Luo Wei. The Integration of Traditional Radio and Internet Radio [J]. Press Outpost,2014-01-15

[10] Ma Hong. Brief Discussion onthe Campus Radio Construction [J]. Private Technology, 2014-06-20

[11] Wang Chengliang. The Analysis of the Current Situation and Development of College Campus Media [J], Southwestern University of Finance and Economics, 2014-04-01 